\title{
Mining a database of single amplified genomes from Red Sea brine pool extremophiles-improving reliability of gene function prediction using a profile and pattern matching algorithm (PPMA)
}

\author{
Stefan W. Grötzinger ${ }^{1}$, Intikhab Alam ${ }^{2}$, Wail Ba Alawi ${ }^{2}$, Vladimir B. Bajic ${ }^{2}$, Ulrich Stingl ${ }^{3}$ and \\ Jörg Eppinger ${ }^{1 *}$ \\ ${ }^{1}$ Division of Physical Sciences and Engineering, KAUST Catalysis Center, King Abdullah University of Science and Technology, Thuwal, Kingdom of Saudi Arabia \\ 2 Division of Biological Sciences and Engineering, Computational Bioscience Research Center, King Abdullah University of Science and Technology, Thuwal, \\ Kingdom of Saudi Arabia \\ ${ }^{3}$ Division of Biological Sciences and Engineering, Red Sea Research Center, King Abdullah University of Science and Technology, Thuwal, Kingdom of Saudi Arabia
}

\section{Edited by:}

R. Thane Papke, University of Connecticut, USA

Reviewed by:

William D. Orsi, Woods Hole

Oceanographic Institution, USA

James A. Coker, University of

Maryland, University College, USA

*Correspondence:

Jörg Eppinger, Division of Physical Sciences, KAUST Catalysis Center, King Abdullah University of Science and Technology, PO Box 2011,

Thuwal 23955-6900, Kingdom of Saudi Arabia

e-mail: jorg.eppinger@kaust.edu.sa
Reliable functional annotation of genomic data is the key-step in the discovery of novel enzymes. Intrinsic sequencing data quality problems of single amplified genomes (SAGs) and poor homology of novel extremophile's genomes pose significant challenges for the attribution of functions to the coding sequences identified. The anoxic deep-sea brine pools of the Red Sea are a promising source of novel enzymes with unique evolutionary adaptation. Sequencing data from Red Sea brine pool cultures and SAGs are annotated and stored in the Integrated Data Warehouse of Microbial Genomes (INDIGO) data warehouse. Low sequence homology of annotated genes (no similarity for $35 \%$ of these genes) may translate into false positives when searching for specific functions. The Profile and Pattern Matching (PPM) strategy described here was developed to eliminate false positive annotations of enzyme function before progressing to labor-intensive hyper-saline gene expression and characterization. It utilizes InterPro-derived Gene Ontology (GO)-terms (which represent enzyme function profiles) and annotated relevant PROSITE IDs (which are linked to an amino acid consensus pattern). The PPM algorithm was tested on 15 protein families, which were selected based on scientific and commercial potential. An initial list of 2577 enzyme commission (E.C.) numbers was translated into 171 GO-terms and 49 consensus patterns. A subset of INDIGO-sequences consisting of 58 SAGs from six different taxons of bacteria and archaea were selected from six different brine pool environments. Those SAGs code for 74,516 genes, which were independently scanned for the GO-terms (profile filter) and PROSITE IDs (pattern filter). Following stringent reliability filtering, the non-redundant hits (106 profile hits and 147 pattern hits) are classified as reliable, if at least two relevant descriptors (GO-terms and/or consensus patterns) are present. Scripts for annotation, as well as for the PPM algorithm, are available through the INDIGO website.

Keywords: bioinformatics, single amplified genomes, halophiles, extermophile, protein sequence consensus patterns, PROSITE IDs, GO-terms, functional genomics

\section{INTRODUCTION}

Discovery of extremophilic enzymes has developed into a major driver for the biotech industry. Although many industrially relevant enzymes were isolated from organisms growing at high temperature, high salt concentration, or in environments contaminated with organic solvents, significant challenges and limitations exist for bio-prospecting of extremophilic enzymes (Liszka et al., 2012). It was estimated that only as few as $0.001-$ $0.1 \%$ of microbes in the seawater are currently cultivatable (Amann et al., 1995) and until recently the bottleneck of cultivation not only biased the view of microbial diversity but limited the appreciation of the microbial world in general (Hugenholtz and Tyson, 2008). Novel culture-independent techniques allow the identification of thousands of novel protein motifs, domains and families from different environments (Yooseph et al., 2007). Despite the vast expectations, metagenomic data have not yet lead to the expected boost of biotechnology (Chistoserdova, 2010), mostly because they suffer from short read length, a low probability to identify rare populations (below 1\%) (Kunin et al., 2008), and difficulties in assembling larger contigs of genetic material for members of complex communities. Single-cell genomics (Lasken, 2007) circumvents this problem, and larger contigs from uncultured organisms can be analyzed. A major challenge in mining genomic data of uncultured organisms is a lack of homology to genes of established organisms resulting in limited reliability of gene annotation. 
A promising source of novel organisms are the deep-sea anoxic brine pools in the northern part of the Red Sea, formed by tectonic shifts (Gurvich, 2006). Interstitial brine was expulsed due to tectonic movements that allowed re-dissolution of evaporitic deposits, and/or phase separation due to temperature variations (Cita, 2006; Hovland et al., 2006). The salt-enriched waters drifted to the seafloor and accumulated in geographical depressions where the brine pools remain stable because of their high density (DasSarma and Arora, 2001). The combination of different extreme physicochemical parameters makes the deep-sea anoxic brine pools one of the most remote, challenging and extreme environments on Earth, while remaining one of the least studied (Antunes et al., 2011). The Red Sea brine pools are extreme in salinity and show a characteristic sharp brine-seawater interface with steep gradients of dissolved $\mathrm{O}_{2}$, density, $\mathrm{pH}$, salinity, and temperature (Emery et al., 1969; Ross, 1972; Anschutz and Blanc, 1995). Except for the connected brine pools Atlantis II, Chain, and Discovery Deep (Backer and Schoell, 1972; Faber et al., 1998), environmental conditions vary drastically between the pools, e.g., temperatures range from $22.6^{\circ} \mathrm{C}$ (Oceanographer) to $68.2^{\circ} \mathrm{C}$ (Atlantis II) and the $\mathrm{NaCl}$ concentration vary from $2.6 \mathrm{M}$ (Suakin) to 5.6 M (Discovery) (Antunes et al., 2011). While the brine pools were detected more than 65 years ago by the Swedish RV Albatross expedition (1947-1948) (Bruneau et al., 1953), microbiological analysis did not start until the late 1960's. The first sampling led to the assumption that under the harsh environmental conditions of the brines life is not possible (Watson and Waterbury, 1969). The search for life in those extreme habitats continuously intensified after the high scientific and economic potential of halophilic organisms became evident (Karan et al., 2012). Since 2010, several sampling expeditions to the Red Sea brine pools have provided a large amount of genomic data, which are collected and annotated at KAUST within the recently described Integrated Data Warehouse of Microbial Genomes (INDIGO) data ware house (Alam et al., 2013). Data stored in INDIGO will stepwise become publicly available.

Analysis and management of next generation whole genome sequencing (NGS) data utilizes comprehensive package of software applications for assembly of sequence reads, mapping to reference genome, variants/SNP calling and annotation, transcript assembly/quantification, and identification of sRNA (Horner et al., 2010; Garber et al., 2011; Pabinger et al., 2014), yet further improvements are required (Dolled-Filhart et al., 2013). Large-scale annotation of DNA sequences with a low homology to genes of experimentally verified function may be flawed and hence represents a major drawback for biomining. The homology-based annotation faces one intrinsic issue: annotation reliability and protein diversity are reciprocal. The situation is complicated by error propagation. The function of the encoded protein was validated experimentally only for a small and continuously diminishing fraction of the gene sequences available. Initially, functions of novel genes were annotated based on gene sequences with experimentally verified function. Based on these data more genes were annotated and so on. While in this chain two proteins are always highly similar, the last annotated gene and the experimentally verified source may possess distinct sequences and functions. In comparison to genomic sequencing, experimental characterization of Single Amplified Genome (SAG) gene products requires gene synthesis, expression, purification as well as functional characterization and therefore is by several orders of magnitude more time consuming. Hence, false positive results from flawed annotation are much more problematic than false negative (due to non-complete annotation) when genomic data are searched for a desired function. This is particularly true for genes from extremophilic organisms, which require slow growing expression systems. Here we present a strategy to minimize false positive identification of the gene product's function. The Profile and Pattern Matching (PPM) algorithm describe below collates complementary information available from (a) InterPro-derived Gene Ontology (GO) terms (Ashburner et al., 2000), which connect an enzyme's function to amino acid sequence profiles and (b) annotated PROSITE IDs (Sigrist et al., 2013), which are linked to an amino acid consensus pattern. This PPM algorithm was tested on 15 protein families of scientific or commercial interest. The strict PPM algorithm initially extracted the most reliably annotated genes, which in this example represent about $1.5 \%$ of the genes in the database. Subsequent removal of incomplete genes followed by PPM selection lead to further condensation of gene hits $(0.1 \%$ of genes in database). A final ranking extracted 11 genes as most likely candidates to code for one of the Protein of Interest (POI) functions.

\section{MATERIALS AND METHODS SAMPLE COLLECTION}

All samples were collected during leg 2 of the RV Aegaeo WHOI, AUC-KAUST Red Sea Cruise in October/November 2011. Samples were taken at different depths and locations in the Red Sea, in and outside the brine pools as well as from sediments. For all brine pools, samples were taken in the brine itself, the sediment and at different depths of the brine seawater interphase (Eder et al., 2001). In total 46 casts were done containing $7030 \mathrm{~L}$ of water, as well as seven sediment samples. The collected liquid samples were immediately filtered using a TFF (tangential flow filtration) system, concentrated and immediately afterwards stored at $-80^{\circ} \mathrm{C}$. During the sampling, different chemical parameters including salinity (conductivity) and temperature were measured. The five brine pools sampled were Kebrit Deep, Nereus Deep, Atlantis II Deep, Discovery Deep, and Erba Deep (Backer and Schoell, 1972; Searle and Ross, 1975; Karbe, 1987; Hartmann et al., 1998).

\section{SINGLE AMPLIFIED GENOME GENERATION}

For the production of SAG from single cells, the "SCGC SAG generation service" (cat. no. S-101) at the "BIGELOW Laboratory single cell genomics center," which is part of the Bigelow Laboratory for Ocean Sciences in Boothbay Harbor, Lincoln County, Maine, United States, was used. The service includes initial sample evaluation for FACS suitability, individual cell separation into wells of a 384-well plate, cell lysis, and single cell multiple displacement amplification (MDA). 


\section{WHOLE GENOME SEOUENCING AND ASSEMBLY}

The whole genome sequencing was performed at the "BIGELOW Laboratory single cell genomics center" using the "Prokaryote SAG whole genome sequencing" service (cat. no. S-014). The service includes sequencing library preparation, genomic sequencing, de novo assembly, and assembly quality control. Service products include contig fasta files and assembly statistics. Assemblies of the single-cell amplified genomes (SAGs) were generated using a pipeline that employs a choice of assemblers designed for single-cell sequencing data including VelvetSC (Chitsaz et al., 2011), SPAdes (Bankevich et al., 2012), and IDBAUD (Peng et al., 2012), along with several pre- and post-assembly data quality checks using Trimmomatic (Lohse et al., 2012). IDBA-UD was benchmarked as the overall best assembler for our SAGs as is it did reconstruct longer contigs with higher accuracy to the reference genome of Nitrosopumilus maritimus SCM1 (Könneke et al., 2005).

\section{DATASET}

The data used in this work consisted of 87 SAGs covering 16 different taxonomic groups, sampled in 11 different environments. A total of 26,626 contigs covering 111,269 ORFs and containing 79.8 Mbp genomic information (Table 1) were analyzed.

\section{Annotation of the dataset}

The assembled contig sequences were integrated into the INDIGO data warehouse (Alam et al., 2013) for microbial genomes. INDIGO is a dynamic system using the InterMine framework (Smith et al., 2012), one of the highest benchmarked data warehouses (Triplet and Butler, 2013). INDIGO allows Automatic Annotation of Microbial Genomes (AAMG), extensive query building for annotation integration, creation of customized feature/attribute/entity lists and enrichment analysis for GO concepts, which are crucial steps of the following analysis. Using INDIGO the assembled contig sequences were (i) annotated, (ii) converted into an XML schema, and (iii) implemented into the data warehouse. Figure 1 gives an overview of the workflow (Alam et al., 2013). Assignments of GO-terms are largely independent from PROSITE IDs. GO-terms emerge from domain associations provided by InterPro (Quevillon et al., 2005) (one of several domain resources may be PROSITE). PROSITE consensus patterns are predicted by the PS_Scan (De Castro et al., 2006) tool.

Automatic annotation of microbial genomes (AAMG) pipeline. Functional annotation of archaeal or bacterial genomes is available via the INDIGO website interface (http://www.cbrc.kaust. edu.sa/indigo/mymine.do?subtab=aamg). Completed genome annotations may be included into the INDIGO database. This enables application of the scripts presented in this work for any novel genetic data.

\section{PHYLOGENETIC ANALYSIS}

The evolutionary history was inferred using the Neighbor-Joining method (Saitou and Nei, 1987). All illustrated trees are drawn to scale, with branch lengths in the same units as those of the evolutionary distances used to infer the phylogenetic tree. The evolutionary distances were computed using the Poisson correction method (Zuckerkandl and Pauling, 1965) and are in the units of the number of amino acid substitutions per site. All positions containing gaps and missing data were eliminated. Evolutionary analyses were conducted in MEGA6 (Tamura et al., 2013).

\section{PPM METHODOLOGY}

The PPM algorithm was automated by including two new scripts into INDIGO, which are publicly available from the homepage.

\section{AutoTECNo: automated translation of E.C. numbers}

The E.C. No. translator (AutoTECNo) automatically converts a list of given enzyme commission (E.C.) numbers into GO-terms (Kanehisa and Goto, 2000) as well as PROSITE IDs, using open source PROSITE files (Sigrist et al., 2002). Preliminary, transferred and deleted E.C. numbers are ignored. The AutoTECNo provides two XML scripts for the independent profile and pattern search via INDIGO. AutoTECNo is available at the following website: http://www.cbrc.kaust.edu.sa/ppma/ec2gops.html.

\section{PPM processor: automated extraction and ranking of the most reliable hits}

The PPM Processor requires one or more tab separated spreadsheets (.tsv) of the independent profile analysis (via GO-terms) and/or pattern analysis (via PROSITE IDs) as input file. The processor generates sets of genes according to their profile and pattern distribution. The resulting list is ranked regarding to the amount of profile and pattern combinations. The PPM processor is available at the following website: http://www.cbrc.kaust.edu. sa/ppma/indigoTbl2PSgoSets.html.

\section{The PPM workflow, starting from a non annotated genome}

First, an assembled genome is annotated using the AAMG pipeline as part of the INDIGO data warehouse. Second the E.C. number based list of POI (list) is translated into profile and pattern values (GO-terms and PROSITE IDs) by using AutoTECNo. The resulting XML lists (of pattern and profile values) are separately imported into the INDIGO

Table 1 | Two example and summary (italic) of the SAG data in INDIGO used for this work.

\begin{tabular}{|c|c|c|c|c|c|c|c|}
\hline Organism & $\begin{array}{l}\text { Habitat } \\
\left({ }^{\circ} \mathrm{C} ; \% \text { salt }\right)\end{array}$ & $\begin{array}{l}\text { Contigs } \\
\text { (number) }\end{array}$ & $\begin{array}{l}\text { Contigs } \\
\text { (min. bp) }\end{array}$ & $\begin{array}{c}\text { Genome } \\
\text { (Mbp) }\end{array}$ & $\begin{array}{l}\text { N50 } \\
\text { (kbp) }\end{array}$ & $\begin{array}{l}\text { N90 } \\
\text { (kbp) }\end{array}$ & ORFs \\
\hline SAR86 clade & $25.0 ; 4.0$ & 86 & 312 & 0.74 & 83 & 6.9 & 853 \\
\hline 87 SAGs, 16 & 11 types & 26,626 & $200-317$ & 79.8 & $1,4-1,200$ & $0.4-13.9$ & 111,269 \\
\hline
\end{tabular}




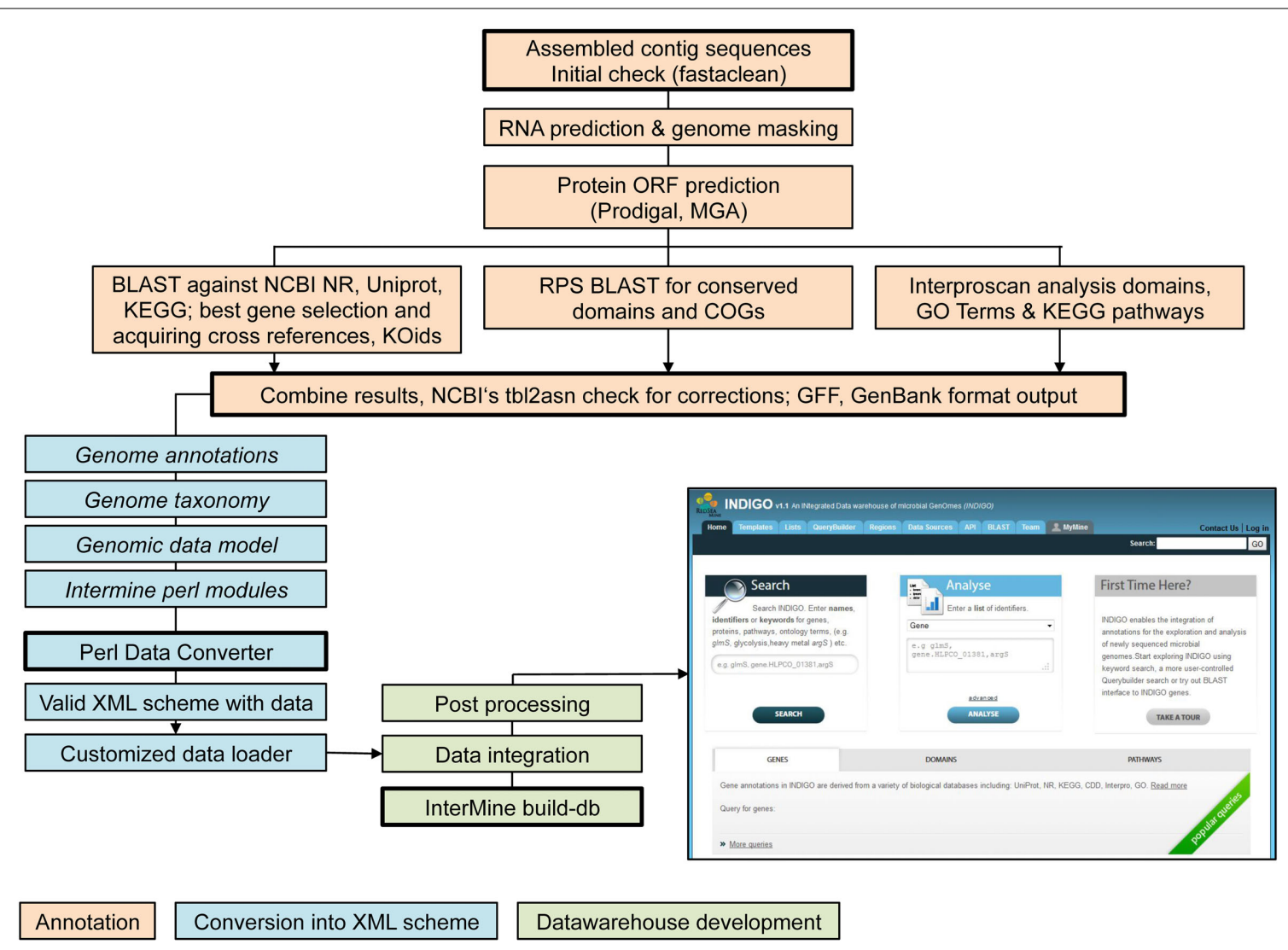

FIGURE 1 | Workflow of data integration into the INDIGO warehouse starting from assembled contig sequences.

data warehouse to analyze any listed genome at the following URL: http://www.cbrc.kaust.edu.sa/indigo/importQueries. do?querybuilder=yes. The two resulting tab separated spreadsheets can be uploaded into the PPM processor to generate three PPM sets of genes: (i) profile set, (ii) pattern set, and (iii) profile and pattern set.

\section{RESULTS \\ PPM: PROFILE AND PATTERN MATCHING FOR FUNCTION IDENTIFICATION}

Analysis of the huge amount of data resulting from next generation whole genome sequencing (NGS) requires modern bioinformatic tools. Comparisons of annotation pipelines reveal a surprising level of uncertainty in gene annotation. Annotations of the same genome (strain TY2482) of the enterohemorrhagic diarrhea causing shiga-toxin-producing E. coli O104 (Rohde et al., 2011) by several groups allowed a comparison of the three main annotation pipelines: Broad, BG7, and RAST. Compared 5164 coding sequences (CDS) of to the Broad annotation the BG7 annotation resulted in 5210 CDS with 163 (3.1\%) false negatives and $271(5.2 \%)$ false positives, and RAST annotation gave in 5446 CDS with $116(2.1 \%)$ false negatives and 321 (5.9\%) false positives (Alam et al., 2013). The AAMG based annotation stored in INDIGO, which is used for this article, gave results similar to those of the Broad institute. Annotation of the E. coli K12 strain W3110 by INDIGO resulted in 4340 CDS (NCBI 4337), with
236 (5.4\%) false positives and 235 (5.4\%) false negatives in comparison to the NCBI annotation. These examples illustrate, that state-of-the-art annotation still yields about 5.5\% false positives for strains of the standard organisms E. coli and a significantly higher rate of false positives may be expected for novel genomes. While this might not impact in silico analysis e.g., for identification of pathways, a substantial amount of false positives can lead to costly failures in experimental bioprospecting campaigns.

Among the descriptors INDIGO annotation associates with genes, two are particularly suited to evaluate the correct assignment of an enzymatic function to a gene product: (i) the GOterm and (ii) the PROSITE ID. The GO project describes genes (gene products) using terms from three structured vocabularies: biological process, cellular component and molecular function. Correspondingly, a list of GO-terms associated with a gene can be seen as the gene's profile. A PROSITE ID relates to a single consensus pattern as "amino acid sequence signature" to characterize protein function. Genes from INDIGO with matching function description of GO-term and PROSITE ID(s) should represent a subset of genes with highly reliable annotation. To extract such genes based on an input list of E.C. numbers of interest, we developed a protein PPM algorithm.

\section{From proteins of interest to bioinformatics descriptors}

Initially, we established a set of proteins, which potentially are of scientific and/or commercial interest. Protein classes selected 
include a variety of hydrolases, ene reductases, dehydrogenases, and carbonic anhydrases (CAs) as well as a range of metalloproteins, porines and potentially new aminoacyl tRNA synthetases. The selected 15 protein families of interest (POI families) are summarized in Table 2. Bioinformatic matching of the POIs vs. the INDIGO database requires a translation of the POI list into terms of the selected descriptors (GO-terms and PROSITE ID). For enzymes, E.C. numbers can be associated with the enzyme family name as well as GO-terms and PROSITE ID and therefore can be used to interconvert these terms. The POI list was translated into the E.C. numbers using BRENDA (Braunschweig Enzyme Database) (Schomburg et al., 2013). Of the resulting 2577 E.C. numbers (Table S1) 434 were non-redundant. Removal of preliminary/transferred and deleted E.C. numbers provided a final list of 265 E.C. numbers (Table S2). The list of E.C. numbers was converted into profiles (GO-terms) and pattern (PROSITE IDs). For gene expression products without enzymatic function like aquaporins and pyltRNA, the respective GO-terms and PROSITE IDs were added manually. The resulting protein profile filter consist of 171 non-redundant GO-terms (BRENDA) (Table S3). The independent pattern filter consisted of 52 non-redundant PROSITE consensus pattern (Sigrist et al., 2013). Three consensus patterns (PS00198, PS00455, PS00143) were removed because of their low specificity (consensus pattern specificity can be derived from the information available at PROSITE web page: http://prosite.expasy. org), resulting in a final pattern list of 49 consensus pattern (Table S4).

AutoTECNo: automated translation of E.C. numbers. The webbased AutoTECNo script simplifies conversion of POI classes into the two bioinformatic PPM descriptors described above. A user may enter one or more distinct or flexible E.C. numbers, which are automatically converted into GO-terms and PROSITE IDs. A numeric value is required for the first three digits of flexible E.C. numbers (e.g., 1.1.1.*). AutoTECNo automatically ignores preliminary, transferred and deleted E.C. numbers. The
AutoTECNo output provides two XML scripts, one for each of the independent profile and pattern search, which can be imported directly into the INDIGO data warehouse by using the direct links on the output page.

\section{The PPM (Profile and Pattern Matching) algorithm}

The PPM algorithm retrieves those POIs from a database, which are most likely to be annotated correctly. Initially, the GOterm list (profile) and the consensus pattern list (coded by the PROSITE IDs) are matched independently onto the dataset of interest. From each of the resulting subset of genomic data, gene fragments commonly present in SAGs or metagenomic data a gene fragment filter eliminates (i) genes with less than 300 nucleotides (to sustain a minimal length required for functionality) and (ii) genes that are not annotated as complete (indicating that a $3^{\prime}$ or $5^{\prime}$ part of the gene is missing). In a last step, both filtered lists are transferred to the PPM processor (see below), which arranges all hits into sets of genes having the same combination of identifiers (GO-terms and/or Prosite IDs). Three classes of sets are listed: (i) the profile sets, containing genes with one or more GO-term describing the respective POI, (ii) the pattern sets, containing genes with one or more PROSITE ID of the respective POI and (iii) the profile and pattern set, consisting of genes with at least one GO-term and PROSIT ID of the POI. The annotation of genes is ranked as more reliable with increasing numbers of associated identifiers. The complete PPM algorithm is illustrated in Figure 2.

Identification of the most reliably annotated genes in INDIGO that match our POI served as test-case for the PPM. The genetic database search was restricted to certain brine pool SAGs based on environmental parameters of the sampling locations (salinity $\geq 14 \%$ and/or a temperature $>44.5^{\circ} \mathrm{C}$ ). The habitats selected were set to reflect the upper part of moderate halophilic conditions (5-20\% salt) as well as extreme halophilic conditions (20-30\% salt) (Ollivier et al., 1994) and/or thermophilic conditions [45-80 ${ }^{\circ} \mathrm{C}$ (Madigan et al., 2003)]. The sample subset comprises 58 SAGs from three different brine pools (Atlantis II

Table 2 | List of proteins of interest (POIs), which were selected for this study.

\begin{tabular}{|c|c|c|c|}
\hline No & POI group & Description & Interest \\
\hline 1 & Alcohol DH & Interconversion of aldehydes/ketones and alcohols & Biocatalytic synthesis of chiral intermediates \\
\hline 2 & Formate DH & Conversion of $\mathrm{CO}_{2}$ into format & Biological carbon capture \\
\hline 3 & Formaldehyde DH & Interconversion of formaldehyde and formate & Biological carbon capture, methanol conversion \\
\hline 4 & Carbon monoxide DH & Interconversion of $\mathrm{CO}$ and $\mathrm{CO}_{2}$ & Biological carbon capture, metalloenzyme structures \\
\hline 5 & Ene reductase & Stereoselective reduction of alkenes & Biocatalytic synthesis of chiral intermediates \\
\hline 6 & Protease & Hydrolysis of peptide bonds & Detergents, food, and leather processing \\
\hline 7 & Terpene synthase & Synthesis of basic, (mulit-)cyclic terpene structures & Biocatalytic synthesis of complex intermediates \\
\hline 8 & Nitrogenase & Fixation of nitrogen from air & metalloenzyme structure and function \\
\hline 9 & Lipase & Hydrolysis of triglyceride esters & Detergents, biodiesel synthesis \\
\hline 10 & Carbonic anhydrase & Interconversion of $\mathrm{CO}_{2}$ and Bicarbonate & Biological carbon capture, metalloenzyme structures \\
\hline 11 & Acetylene hydratase & Synthesis of aldehydes from acetylene & Biocatalytic synthesis intermediates \\
\hline 12 & Acetyl-CoA synthetase & Activation of acetate for further conversion & Biological carbon capture metabolism \\
\hline 13 & pylRS & Aminoacyl tRNA synthetase, acting on pyrrolysine & Synthetic biology, expanding the genetic code \\
\hline 14 & pyltRNA & tRNA coding for pyrrolysine & Synthetic biology, expanding the genetic code \\
\hline 15 & Aquaporin & Integral membrane proteins controlling osmotic pressure & Water desalination membranes \\
\hline
\end{tabular}




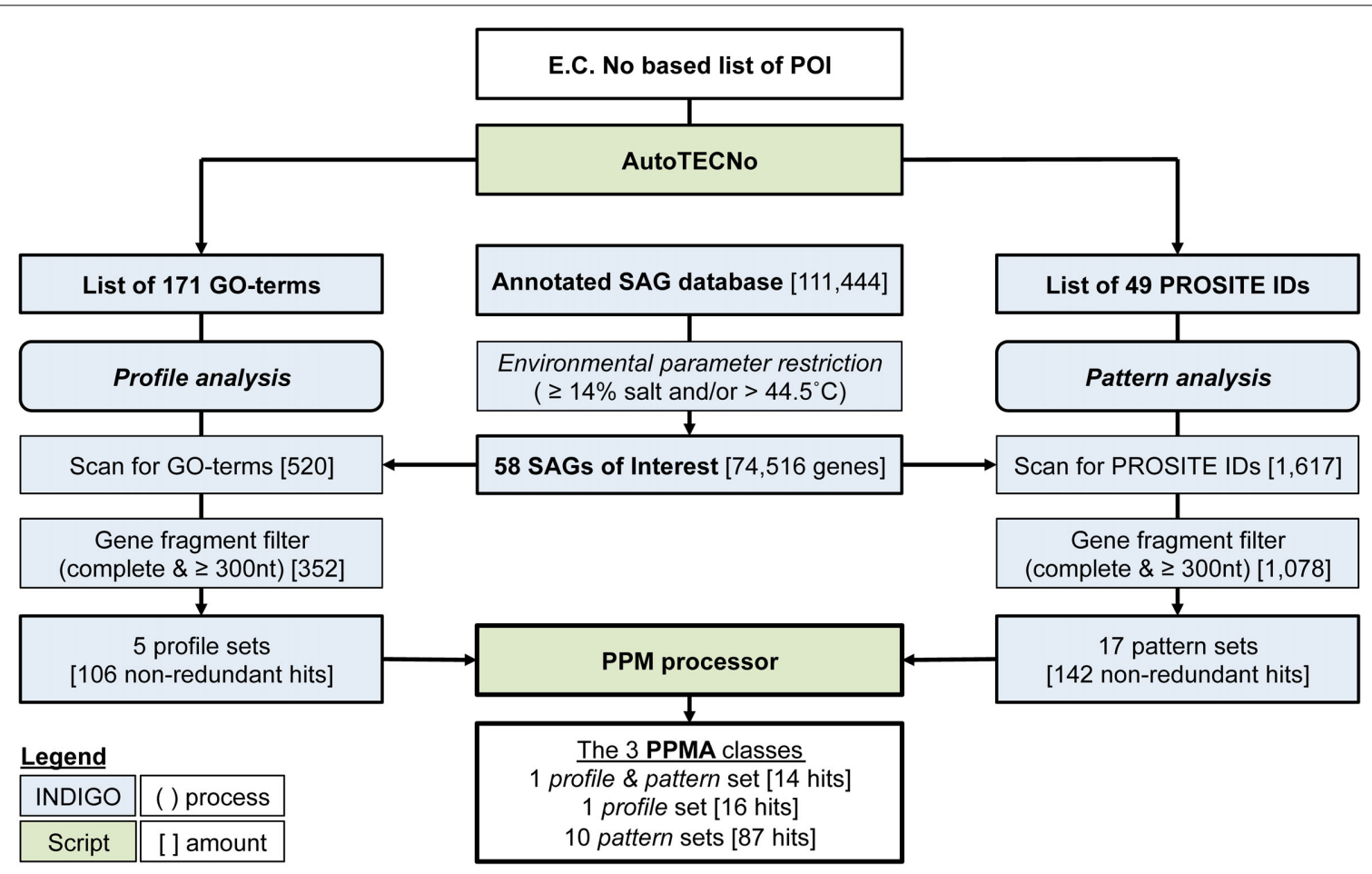

FIGURE 2 | Flowchart illustrating the PPM (profile pattern matching) algorithm, starting from an E.C. Number based proteins of interest (POI) list and a selected database subset, which may also be uploaded externally. Numbers refer to the example published here. Square brackets indicate number of genes at each step during the example analysis, specific restriction filters are described in normal brackets. The complete PPM algorithm is available at the INDIGO webpage including the scripts AutoTECNo and PPM Processor. deep, Discovery, and Kebrit), covering six different environmental conditions. These SAGs contain a total of 73,688 ORFs coding for 74,516 genes. The ORFs were assembled out of 21,519 contigs into genomes of a combined size of 48.2 mega base pairs (Table 3 ).

As described above, the POI list was transformed into a protein profile filter consisting of 171 non-redundant GO-terms (BRENDA) and an independent pattern filter of 49 PROSITE IDs (Sigrist et al., 2013). Profile matching of the 74,516 preselected genes with the 171 GO-terms resulted in 520 hits, which were further reduced by the gene fragment filter to 352 (Table 4). Elimination of duplicates (genes associated to multiple GO-term or PROSITE ID occur multiple times in the output) yielded 106 non-redundant hits, which could then be grouped into five different profile sets, based on the gene-associated GO-terms. The five profile sets contain six different GO-terms, four profiles with only one GO-term and one profile with two GO-terms (Table 5). Categorizing the 106 genes into five profile sets clarifies what functions and functional diversity can be expected from the hits.

The independent pattern filter was applied according to the same scheme. Screening all 58 SAGs against the 49 PROSITE IDs resulted in 1617 hits. Applying the gene reliability filter reduced this number to 1078 hits, which could be further condensed to 142 non-redundant hits. These 142 genes fall into 17 pattern sets containing 25 different PROSITE IDs.

Since the presence of several GO terms, PROSITE IDs or a combination of both indicates a more reliable gene annotation, we used the PPM processor to identify genes which are associated with multiple descriptors. The list (Table 5) contains three sub-sets: (i) the profile sets (one set of 16 hits), (ii) the pattern sets (10 sets containing 87 hits), and (iii) the profile and pattern sets (one set of 14 hits). Only the profile and pattern set contains genes, which were found independently by both, PPM. In other words, when the INDIGO subset of 74,516 genes is screened for the 434 non-redundant E.C. numbers, only 14 genes have a matching GO-term and PROSITE ID. All 14 hits belong to the same E.C. number (1.3.1.26, dihydrodipicolinate reductase, DHPR). Since some profile or pattern sets stand for the same enzyme type the total amount of 117 most reliably annotated genes that were identified by the PPM algorithm fall under only nine different enzyme families: prephenate DH (1.3.1.13), iron containing ADH (1.1.1.1), dkgA (1.1.1.274), glyoxylate reductase (1.1.1.26), Clp protease (3.4.21.92), molybdopterin oxidoreductase (e.g., 1.2.2.1), nitrogenase (1.18.6.1), subtilisin $\left(3.4 .21 .^{*}\right)$, and DHPR (1.3.1.26). The relatively small number of highly reliable hits is helpful for an experimental scientist, who is aiming to characterize novel gene expression products. A reduction of 111,444 potential expression targets to only 117 provides the necessary experimental focus (see below).

Semi-automatic, XML based PPM algorithm. The PPM algorithm was integrated into the INDIGO web page via a XML script. The semi-automated work flow requires three steps: (i) conversion of the POI list into GO-terms and PROSITE IDS with 
Table 3 | Bacterial (italic) and archeal SAGs from thermophilic and hypersalinic sampling regions selected for this study.

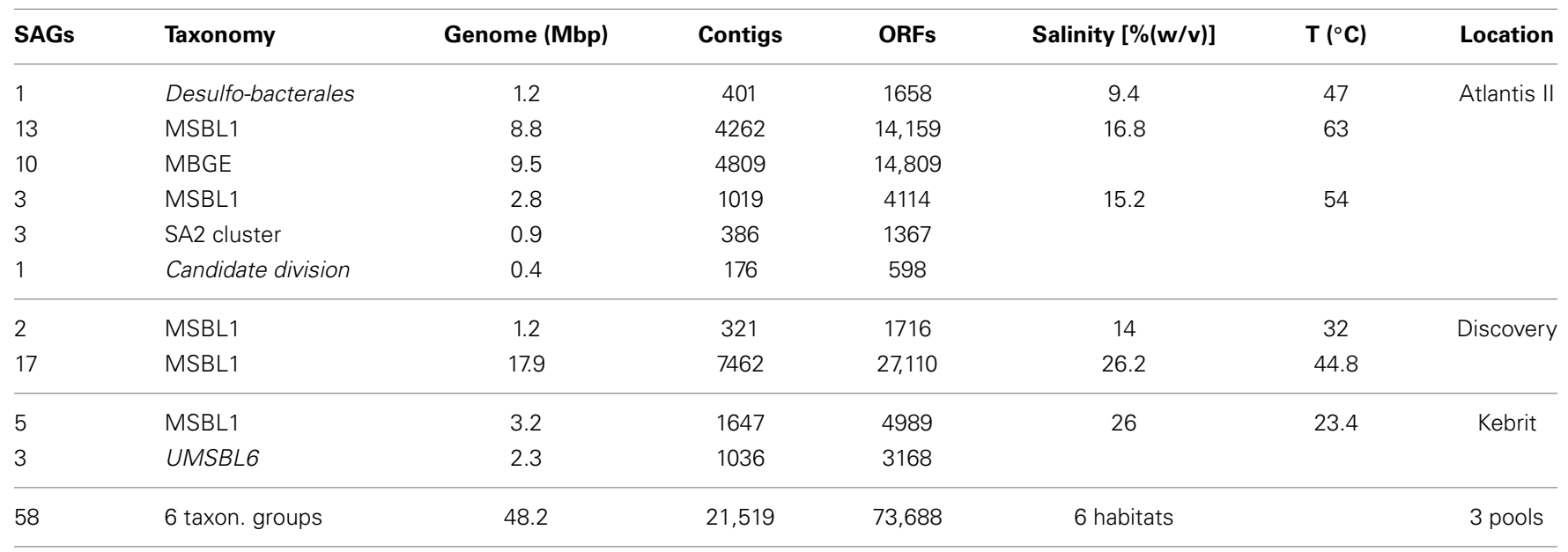

Table 4 | Stepwise overview of the conversion the 15 selected enzyme groups into non-redundant GO terms and PROSITE ID.

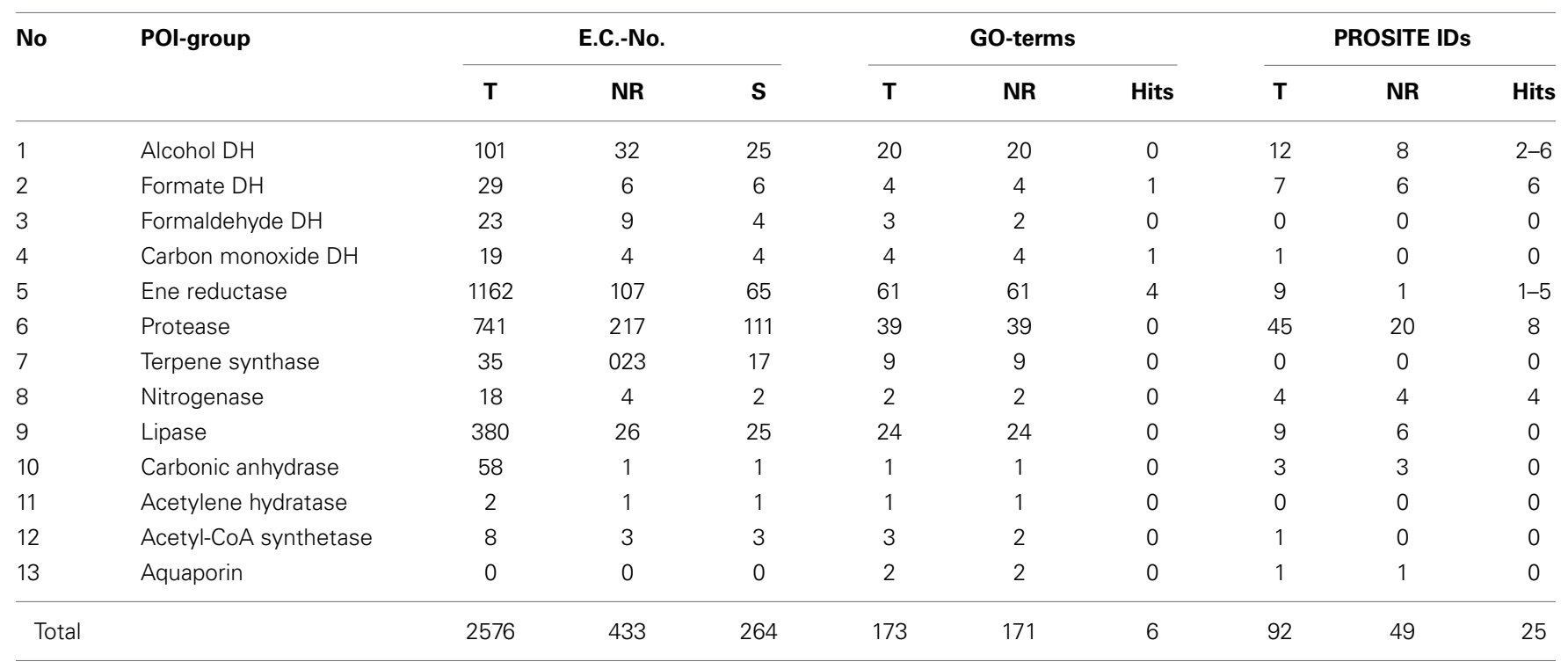

T, Total in class; NR, non-redundant ones; S, selected for this study.

AutoTECNo, (ii) individual profile as well as pattern matching via a query in INDIGO and (iii) extraction and ranking of the most reliable result in pattern, profile and profile and pattern by the PPM processor. This process requires two input files: (i) an assembled genome, which can be annotated using the AAMG pipeline and (ii) an E.C. number based POI list. The POI list can directly be copied into the AutoTECNo input mask. After submitting the E.C. number list, AutoTECNo will generate a list of all E.C. numbers and the associated GO-terms and PROSITE IDs. At the bottom of the output mask, three links are provided: "GO xml," "Prosite xml," and "INDIGO datawarehouse." Clicking either of the first two links will open a window, which provides.xml-formatted files (for either GO-terms or PROSITE IDs). These files can be edited and used separately to build INDIGO queries. In such a query, INDIGO is used to match each of the two.xml lists against the selected genomes. Clicking on the "INDIGO datawarehouse" link opens the INDIGO XML input mask, which can be used to initiate a query by pasting the.xml script from AutoTECNo. A graphical overview of the query will be shown and further customization can be done (preset columns should not be deleted). At this stage, both, profile (GO-term) and pattern (Prosite ID) filters can be applied individually in connection with the optional gene fragment filter. Hits will be organized in a table summarizing all information available in INDIGO. The table still may contain duplicates, since one gene can be found under several GO-terms and/or PROSITE IDs. The results-table can be downloaded as "Spreadsheet (tab separated values)" (.tsv file) for import into the PPM processor. The PPM processor output provides a list of non-redundant genes, grouped into subsets of the three classes of hits (profile sets, pattern sets and profile and pattern sets) as well as ranked based on the amount of associated patterns and profiles. A link back to INDIGO allows listing of the obtained hits for a detailed analysis. 
Table 5 | Enzyme hits identified using the PPM algorithm and the respective PPM descriptors.

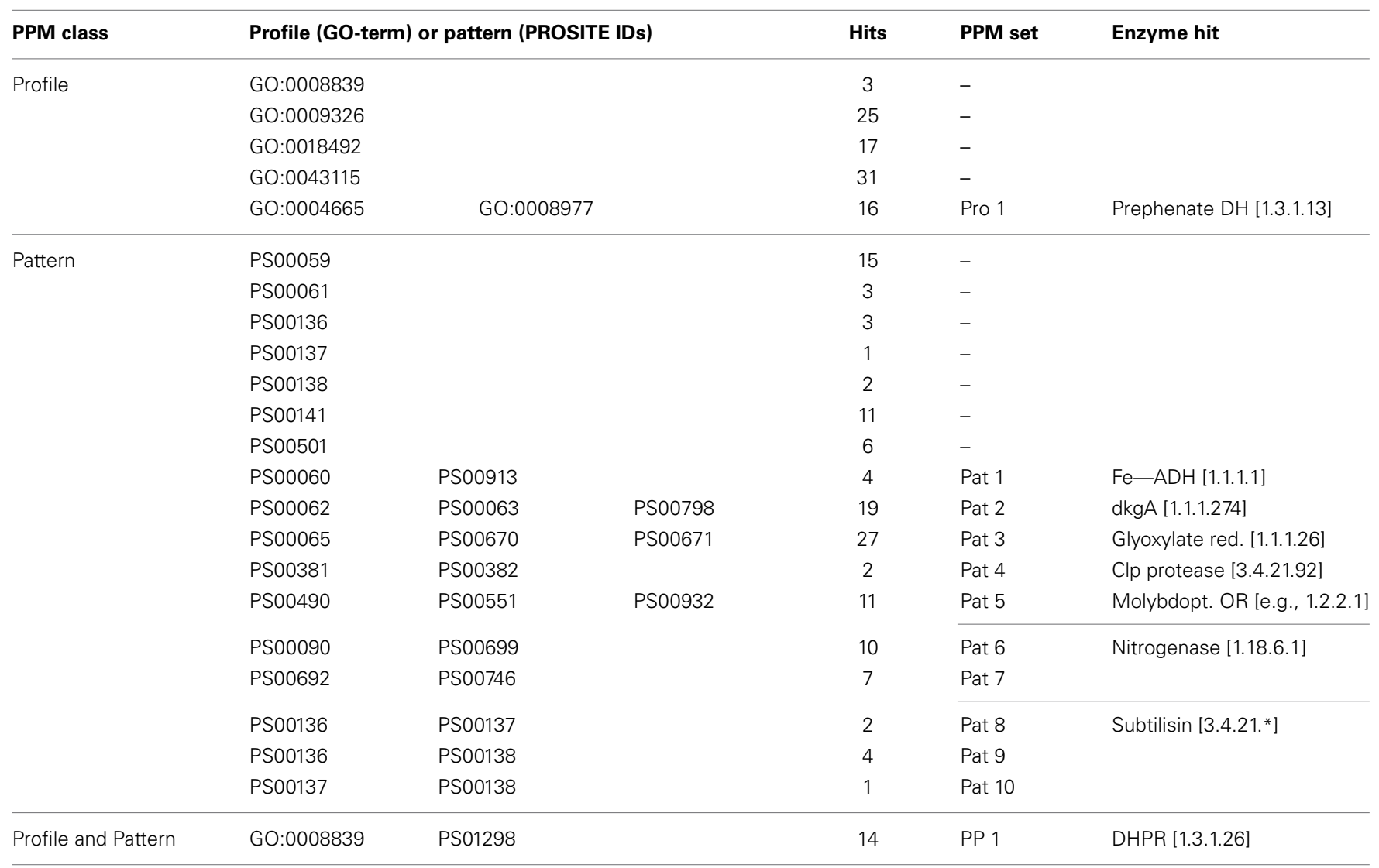

\section{MANUAL HIT SELECTION FROM THE PPM PROCESSOR OUTPUT}

Grouping of genes into PPM classes and sets immediately highlights expected functional similarities of gene expression products. PPM sets of patterns and/or profiles, which are characteristic for the same protein, can be condensed further into one metaset. For example pattern sets with combinations of PS00136 and PS00137, PS00136, and PS00138 or PS00137 and PS00138 are all indicative of subtilase type serine proteases and these pattern sets were condensed into one meta set. In total nine functionally distinct PPM sets remained after manual condensing (Table 5).

For experimental characterization, synthesis and expression of 117 genes from halophilic extremophiles still represent an enormous challenge, which mandates identification of those extremophilic proteins as expression targets, which are most typical for each functionality-set. For five of the nine functionally different PPM sets, we were able to pinpoint nine genes representing all three PPM classes (profile, pattern, profile, and pattern) (Table 5). Amino acid based phylogenetic analysis within each PPM set revealed phylogenetic relations and sequence clusters. The sequence representing most of the set-members was selected, e.g., the PP1 DHPR PPM set contains 14 different hit sequences (isoenzymes). Phylogenetic analysis resulted in four clusters of phylogenetic closely related groups (Figure 3). For each of those four clusters the sequence representing most of the members was selected. This was straightforward for three DHPR clusters, since one sequence contained all elements of the others. In the fourth case as well as for cluster of other sets the selection was more complicated, because phylogenetic sequence clusters showed either an equal distribution of mutations in one cluster or an unequal length of sequences. To address this problem an additional protein BLAST (BLASTp) (Johnson et al., 2008) was performed and the sequence with the highest similarity was chosen for the fourth DHPR and the halolysin cluster. In case of no difference in similarity according to BLASTp, the gene product providing more functional side chains was chosen (e.g., for subtilisin) since additional chemical functionality may indicate more diverse enzyme characteristics (e.g., hydrogen bonding, allosteric pockets, metal complexation etc.). Amino acid sequences typically differed in less than 10 positions [amino acid sequence length: 401 (ADH), 348 (2-hydroxyacid DH), 498-565 (halolysin; the 565 amino acid sequence contains all shorter ones), 528 (subtilisin), 435-440 (prephenate DH), 272-285 (four subgroups of DHRPs)].

\section{FUNCTION IDENTIFICATION OF PROTEINS WITHOUT EXISTING GO-TERMS OR PROSITE IDS}

The initial search for CAs was not successful. While distinct GOterms and consensus patterns exist for $\alpha$ - and $\beta$-CAs (Table 5), non are available for the other three CA families $(\gamma, \delta$ and $\zeta)$. According to Ferry the CS chain A from Methanosarcina thermophile (Cam) can be considered the archetype of the $\gamma$-CA family, and a distinct, 180 amino acid sequence (no 34-214) is indicative for a $\gamma$-CA protein (Smith and Ferry, 2000). An 


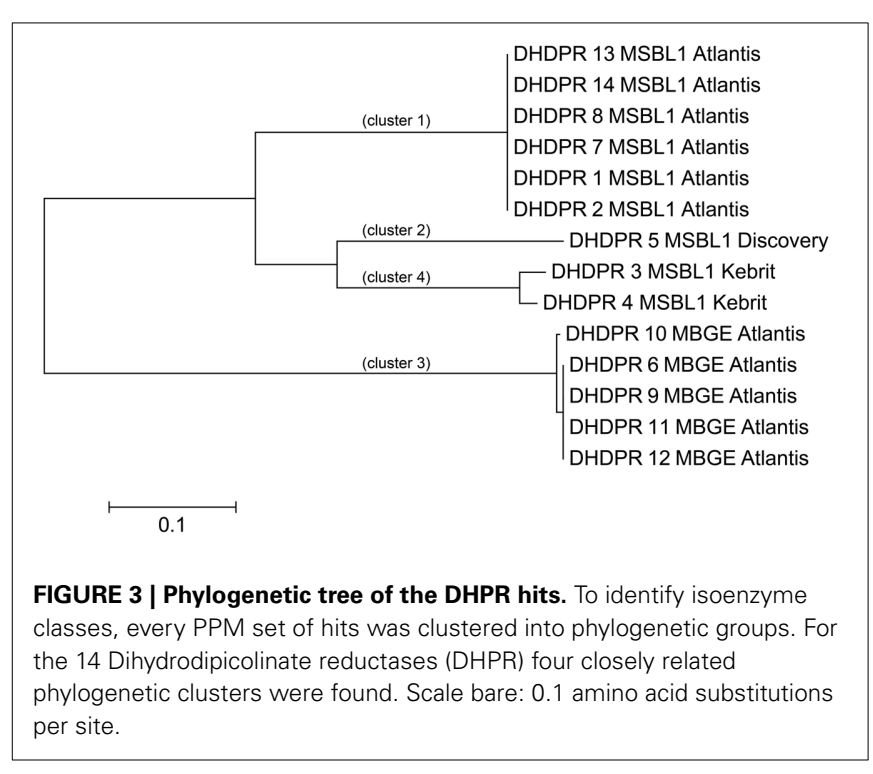

INDIGO internal BLAST of this 180 amino acid motif against all genes yielded 17 potential $\gamma$-CAs. Applying the gene fragment filter reduced the candidate pool to six.

As discussed above, an additional pattern matching should increase the reliability of the profile-based protein identification. The analysis of the only two $\gamma$-CA class crystal structures reported (Cam from M. thermophila Kisker et al., 1996, see also pdb 3OW5 and a CamH homolog from P. horikoshii Jeyakanthan et al., 2008) revealed nine amino acids in two peptide sequences of 26 and six amino acids as most relevant for enzyme function (Smith and Ferry, 2000). The resulting two initial consensus patterns are shown below [color code: yellow, metal binding motifs $\left(\mathrm{H}_{81}\right.$, $\left.\mathrm{H}_{117}, \mathrm{H}_{122}\right)$; green, residues directly involved in catalysis $\left(\mathrm{E}_{62}\right.$, $\left.\mathrm{N}_{73}, \mathrm{Q}_{75}, \mathrm{E}_{84}\right)$; blue, structurally important residues $\left(\mathrm{R}_{59}, \mathrm{D}_{61}\right)$; not highlighted, residues of no specific function as they appear in the $\gamma$-CA sequence].

$59-\mathrm{R}_{59} \mathrm{SD}_{61} \mathrm{E}_{62} \mathrm{GMPIFVGDRSN}_{73} \mathrm{VQ}_{75} \mathrm{DGVVLH}_{81} \mathrm{ALE}_{84}$ 84 and

$117-\mathrm{H}_{117} \mathrm{QSQVH} \mathrm{H}_{122}$ - 122

No hit was found for a strict pattern matching of the six potential $\gamma$-CAs. This is not surprising, since it is common for consensus patterns that some functionally important amino acids can be altered within a certain threshold. Alignment of the initial $\gamma$-CA consensus pattern with the six $\gamma$-CA candidate sequences revealed that the 10 amino acid long stretch from $\mathrm{E}_{62}$ to $\mathrm{N}_{73}$ was shortened by one amino acid in all six candidates. The resulting structural alteration is unlikely to affect function. Further, the two structurally important residues $\mathrm{R}_{59}$ and $\mathrm{D}_{61}$ were conserved as well as two out of the three metal binding histidines $\left(\mathrm{H}_{81}\right.$ and $\left.\mathrm{H}_{117}\right)$ (Table 6). The third metal binding amino acid $\mathrm{H}_{122}$ was replaced by an $\mathrm{N}$ in hit number 6 , a mutation, which potentially affects function. Further sequence variations involve the replacement of catalytic $\mathrm{E}_{84}$ by either $\mathrm{D}$ (four cases, potentially not influencing function), or $\mathrm{K}$ (two cases, potentially affecting function). The remaining catalytically important residues $\mathrm{E}_{62}$, $\mathrm{N}_{73}$, and $\mathrm{E}_{75}$, which are involved in a hydrogen-bonding network in the M. thermophile protein, are highly variable among the six candidates sequences. Assuming that some of these candidates are CAs because of profile and pattern similarity to the $M$. thermophile archetype enzyme we concluded that $\mathrm{E}_{62}$ is not generally important for the function of this enzyme type and that $\mathrm{N}_{73}$ and $\mathrm{E}_{75}$ can be replaced by the hydrogen bonding amino acids $\mathrm{C}$ or $\mathrm{K}$, respectively. Correspondingly, we suggest the following two consensus patterns for $\gamma$-CAs:

$\mathrm{R}-\mathrm{x}-\mathrm{D}-\mathrm{x}(10,11)-[\mathrm{NC}]-\mathrm{x}-[\mathrm{QK}]-\mathrm{x}(5)-\mathrm{H}-\mathrm{x}(2)-[\mathrm{ED}]$ and

$\mathrm{H}-\mathrm{x}(3)-\mathrm{H}$

Application of the PPM algorithm using the 180 amino acid profile stretch identified from pdb 3OW5 and the new consensus patterns delivered three $\gamma$-CAs candidates. Because of high sequence similarity in two out of the three sequences, the sequences of gene 2 (annotated as ferripyochelin binding protein 01) from Atlantis II deep and gene 3 (annotated as predicted acetyltransferase) from discovery deep (Table 6) were selected as best candidates for experimental studies of $\gamma$-CAs [CA_A (Atlantis II deep) and CA_D (Discovery deep) in Table 7].

\section{DISCUSSION}

Proteins, which are suitable for the harsh conditions of many biotechnological applications can be obtained through protein engineering, discovery and mining of novel extremophilic genomes or a combination of both. The major challenge in mining genomic data from extreme environments is, that, with increasing extremeness of the habitat, the possibility of culturing the organism thriving under these conditions shrinks substantially (Alain and Querellou, 2009). However, SAGs can provide genomic data from uncultured organism. We believe that improving the quality of SAGs assemblies (higher sequence coverage, longer contigs, and advanced annotation programs) should enable us to utilize SAGs as a rich source for discovery of extremophilic enzymes of scientific interest and commercial value. However, annotation reliability is lowered for both, extremophilic genomes (for which commonly no close relative is known) and SAGs (which may suffer from gaps, incomplete genes, or generally sequencing data of lower quality) and therefore a highly reliable algorithm for identification of genes of interest from extremophilic SAG databases is mandatory before entering labor-intensive expression and characterization of these genes.

\section{PROBLEMS OF SINGLE PROFILE OR PATTERN ANALYSIS AND THE PPM ALGORITHM}

Consensus patterns show a good reliability, yet a considerable amount of hits identified via PROSITE ID are false positives (has the motif but not the function), false negatives (has the function but not the motif), unknown (has the motif but no verified function), or partial hits (has the function but only parts of the motif) (Sigrist et al., 2002). Table 8 combines examples illustrating the reliability for consensus pattern based annotation of enzyme function. Reliability may be as low as $55 \%$ false positives (PS00136) or 90\% false negatives (PS00065). A further problem of pattern-based annotation is the low flexibility because of the short pattern lengths (about 10-20 amino acids Sigrist et al., 2002), typically covering only $1.9-7.9 \%$ of the total protein length. Due to the short length of the consensus pattern, a 
Table 6 | Alignment of functional important residues the $\gamma$-CA chain A from Methanosarcina thermophile with $\gamma$-CA candidates.

\begin{tabular}{|c|c|c|c|c|c|c|c|c|c|c|c|}
\hline No. & Annotation & Organism and habitat & $\mathbf{R}_{59}$ & $D_{61}$ & $E_{62}$ & $\mathrm{~N}_{73}$ & $\mathbf{Q}_{75}$ & $\mathbf{H}_{81}$ & $E_{84}$ & $\mathbf{H}_{117}$ & $\mathrm{H}_{122}$ \\
\hline 2 & Ferripyochelin binding protein 01 & MSBL1, Atlantis $\|\left(63^{\circ} \mathrm{C}, 16.8 \%\right.$ salt $)$ & \multirow{4}{*}{\multicolumn{2}{|c|}{ 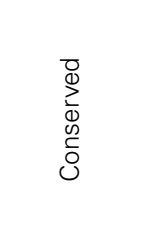 }} & $\mathrm{F}$ & $\mathrm{C}$ & $\mathrm{Q}$ & \multirow{4}{*}{ 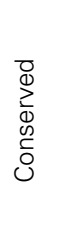 } & $\mathrm{D}$ & \multirow{4}{*}{ 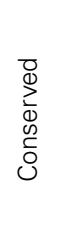 } & \multirow{3}{*}{ 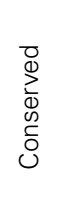 } \\
\hline 4 & Ferripyochelin binding protein 02 & MSBL1, Kebrit $\left(23.4^{\circ} \mathrm{C}, 26.0 \%\right.$ salt $)$ & & & $\mathrm{F}$ & $\mathrm{C}$ & G & & $\mathrm{D}$ & & \\
\hline 5 & Ferripyochelin binding protein 03 & MBGE, Atlantis II $\left(63^{\circ} \mathrm{C}, 16.8 \%\right.$ salt $)$ & & & $K$ & $\mathrm{~N}$ & Q & & K & & \\
\hline 6 & Ferripyochelin binding protein 04 & MSBL1, Discovery $\left(44.8^{\circ} \mathrm{C}, 26.2 \%\right.$ salt $)$ & & & $\mathrm{F}$ & V & $E$ & & K & & $N$ \\
\hline
\end{tabular}

Table 7 | Hits identified as reliable using the PPM algorithm.

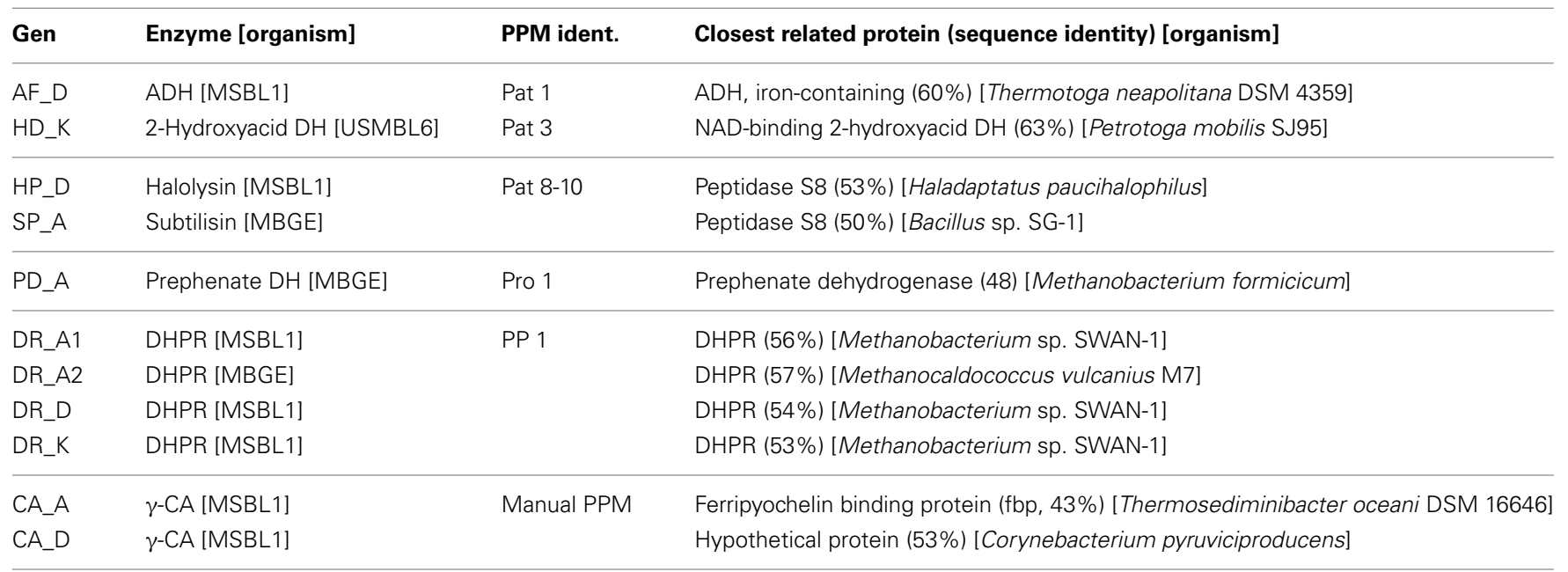

Last letter of gene name indicates habitat: D, Discovery; A, Atlantis II; K, Kebrit.

higher reliability requires reducing the permissible flexibility. In the CAs example above, three consensus patterns were available with high reliability (Table 8). Hence we expected to identify several CAs through pattern matching. Yet, no CA was found in the entire database since the rigidity of these consensus patterns prevented identification of novel enzymes with the same function. Finally, a consensus pattern may not be specific for a specific function, e.g., NADH or ATP binding motifs typically are associated with consensus patterns, which occur in several enzyme families. Table 7 illustrates this issue. Four PROSITE IDs are related to both, either alcohol dehydrogenase or ene reductase function. Identifying combinations of patterns can circumvent these problems and increase reliability. According to the PROSITE web page, one of the strongest pattern combinations is PS00136-PS00138. If a protein includes at least two of the three active site signatures, the probability of it showing a protease activity is assumed to be $100 \%$.

Ontologies are widely used for functional annotation (Radivojac et al., 2013). Gene ontologies are commonly expressed by GO-terms. The source for GO-terms in the UniProt Gene Ontology Annotation database falls into three categories: (i) the smallest but most reliable category, experimental annotations, (ii) curated non-experimental annotations and last electronic annotations, (iii) with less reliability. Over $98 \%$ of the repository of the UniProt Gene Ontology Annotation database is inferred in silico without curator oversight (Škunca et al., 2012). GO-terms are highly flexible, which is reflected in the gene's sequence length associated with it, e.g., annotation of GO-terms in this study covered $1.9-100 \%$ of the total gene. The particular sources used for GO-term identification leads to this large range. GO-terms based on consensus pattern naturally are reflected by a short associated sequence length (e.g., the $1.9 \%$ lower limit in this study). GOterms determined by different methods (e.g., Hamap, TIGRfam, PIRSF) can take up to $100 \%$ of the sequence into consideration. In this analysis GO-terms association to ORFs was in average based on about $65 \%$ of the total sequence length. Recent studies could show that electronic annotations are more reliable than generally believed and that the overall reliability of electronically determined GO-annotations is increasing, but still very low. The mean value of reliability was $\approx 30 \%$ in 2006 and increase to $50 \%$ in 2011 (Škunca et al., 2012). The variations are significant among different inference methods, types of annotations, and organisms. Further, functional annotation, which is only based on GO-terms can result in a considerable bias (Schnoes et al., 2013). INDIGO utilizes all InterProScan derived GO-terms whether they are emerging from longer domains such as PFAM, TIGRfam, or PROSITE short consensus patterns. It is common that PROSITE IDs do not relate to any GO term, yet a longer domain in the vicinity or around a PROSITE pattern yields a GO-term associated to a POI. Currently, 11,910 ORFs (10.6\%) annotated in INDIGO are associated with a GO-term and a PROSIT ID, which both describe the same function. The INDIGO data warehouse 
Table 8 | Reliability of consensus patterns found in chosen hits as well as for carbonic anhydrases.

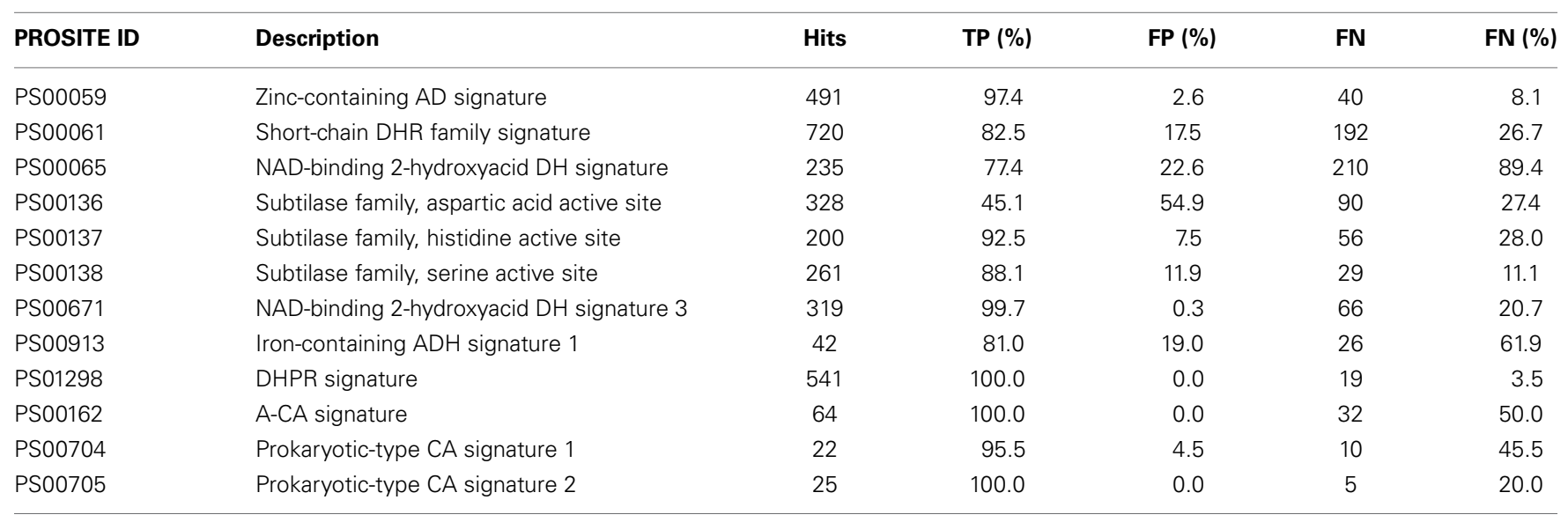

TP, True positive; FP, False positive; FN, False negative (Sigrist et al., 2002).

based annotation (AAMG) combines various annotation methods. Unlike other data warehouses, INDIGO keeps and organizes all annotation meta data even if these are not in agreement with the final annotation (Alam et al., 2013). All GO-term and PROSITE IDs, which are available from these meta data are used by the PPM algorithm. In two cases, the PPM algorithm based function predictions differ from the INDIGO annotation. $\gamma$-CA identified by the PPM algorithm were previously annotated as "predicted acetyltransferase isoleucine patch superfamily" or "Ferripyochelin binding protein." Other PPM algorithm based functions narrowed the INDIGO annotation down to only one function. The prephenate DHs were originally annotated as both, Chorismate and Prephenate DH.

In summary both, consensus patterns and GO-terms are standard tools to identify the function of a gene, yet they have weaknesses. The key to increase reliability is combination of descriptors. Since GO-terms (profiles) and PROSITE IDS (patterns) provide orthogonal information of protein function (with the exception of GO-terms based on consensus patterns) selecting combination of both descriptors is a powerful tool to identify the function of a gene product with higher reliability, particularly for novel and distantly related organisms. The PPM algorithm combines those advantages and is able to select for all three combinations of descriptors: the profile sets, the pattern sets and the profile and pattern sets. The strict PPM algorithm extracts and ranks in our case the top $0.1 \%$ of most reliably annotated genes. Since genomic data are growing at a much faster pace than experimental verification can proceed, a focus on quality rather than quantity is required. The PPM algorithm guides experimentalists to relevant starting points for successful expression, characterization, and verification of gene products.

\section{DISTANTLY RELATED SEQUENCES FROM NOVEL ORGANISMS}

Phylogenetic analysis of gene sequences identified as candidates for expression tests revealed a high evolutionary distance to any known sequence (Figure 4). In case of the PPM profile and pattern set hits, which all are DHPRs, the phylogenetic tree with the closest related organisms includes both, the archeal and bacterial domains of life (Figure 4A). The four identified hits are all in the archeal branch. The three hits from the organism MSBL1 (DR_A1, DR_D, and DR_K) are clustering together in a separate branch, connected to Acheoglobales and Methanomicroba. The hit from the organism MBGE (DR_A2) is in a separate branch and closer related to Methanobacteria and Methanococci. As indicated by the long branches the junction to the closest previously known sequences occurs at $0.3-0.35$ amino acid substitutions per site. The PPM multi-profile hit prephenate dehydrogenase from MBGE (Figure 4B) shows phylogenetic relations similar to DHPR. The closest related enzymes found are from archea and the closest related sequences are from Methanococci and Methanobacteria. The junction to the closest previously known sequences occurs at 0.33 amino acid substitutions per site. The subtilase type sequence from the PPM multi-pattern hit has a different phylogenetic footprint (Figure 4C). Based on the amino acid sequence the novel subtilisin shows equal evolutionary relations to archea and bacteria, which indicates comparatively low sequence mutations in the two different domains compared to their common ancestor. For the $\gamma$-CA hits, which are based on a combination of a new profile and pattern, the phylogenetic tree includes all three known classes of CA (Figure 4D). The tree reveals clearly, that the identified sequences fall into the $\gamma$ class of CAs with very distant relations to the $\alpha$ and $\beta$ class. Distant phylogenetic relationships are also found for all other hits, underlining the novelty of the SAGs analyzed (Figures S1-S3).

\section{CURRENT LIMITATIONS OF THE PPM APPROACH}

The PPM approach intrinsically leads to a high number of false negatives, because not all protein of interest groups can be translated into GO-terms and PROSITE IDs. During conversion from E.C. numbers to profiles (GO-terms) or pattern (PROSITE ID) about 35 or $81 \%$ of the POIs are lost, respectively. This limitation will be overcome through the exponential growth of biological data, which will increase the number and precision of GO-terms and PROSITE IDS. The combination of self-derived profiles and pattern can also enhance/enable PPM analysis, even with comparatively flexible sequences that show individually low reliability, as shown for the $\gamma$-CA example. Reducing the rigidity of consensus pattern with a high false negative rate may further help to increase 


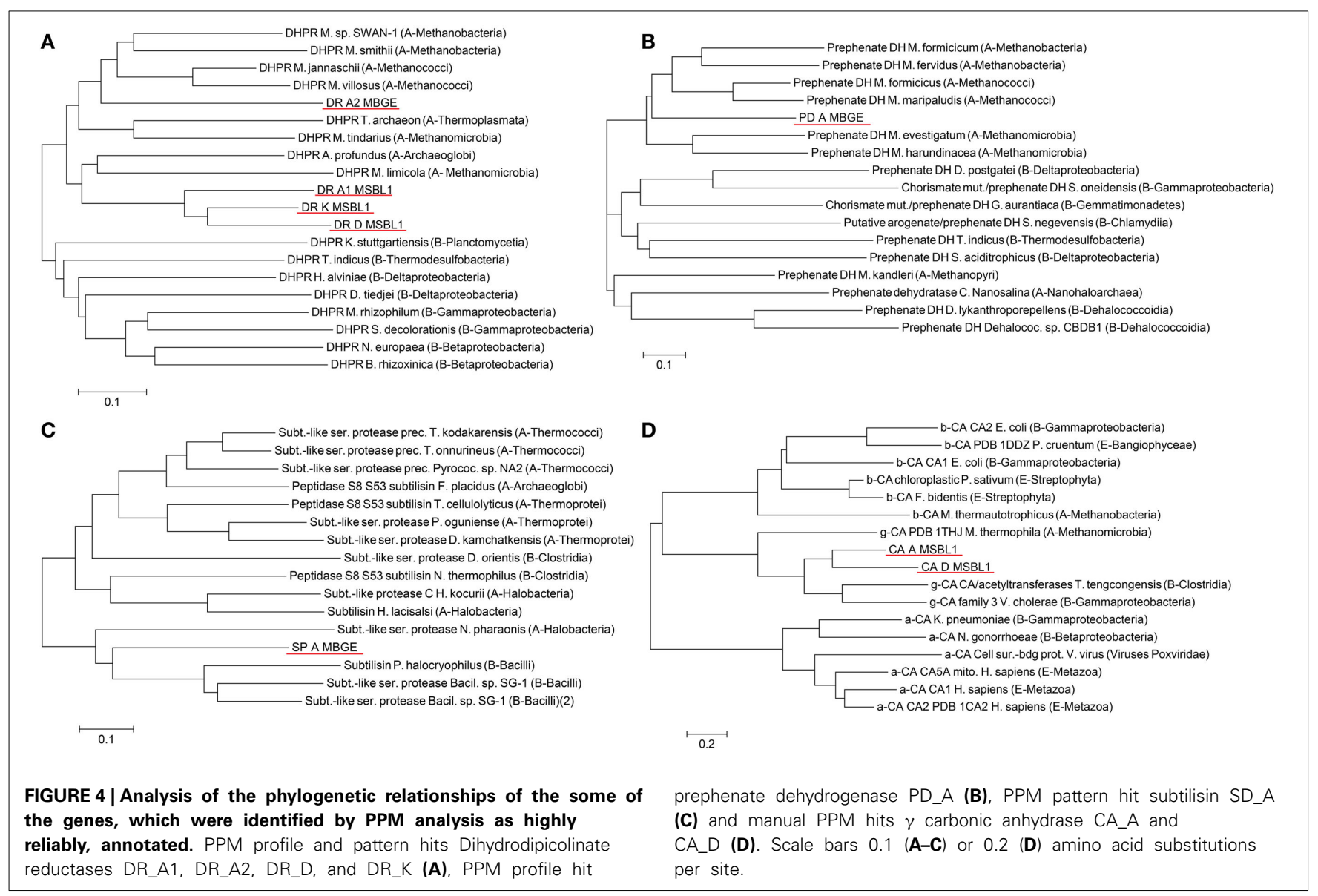

hit rates. However, as discussed above, from an experimentalist point of view false positives are of much higher concern and these can be eliminated very effectively by the PPM approach.

\section{OUTLOOK AND CONCLUSION-THE RED SEA EXTREMOPHILES AS SOURCE FOR NOVEL ENZYMES WITH HIGH SCIENTIFIC AND INDUSTRIAL POTENTIAL}

For the first time SAGs were used to identify proteins for biotechnological applications. The eleven different genes, which were extracted from the INDIGO database during this study as candidates for expression just give a glimpse of the potential the Red Sea brine pools extremophiles have for discovery of novel enzymes. Not only the great phylogenetic distance to any described organism but also the extreme anoxic, high temperature, and hypersaline environment makes the enzymes of those organisms highly valuable. Enzymatic activity at high temperature and with low water activity can enable biocatalysis to be a tool for complex chemical reactions giving high yield and enantiomeric excess and under conditions that were so far out of reach for biological applications. Investigation of the enzymes, for which genes were identified here, will help understanding the limitations and adaptation of life at such extreme places.

The PPM algorithm is not intended to be a competitor for standard annotation. However, it is a powerful tool to analyze functions of proteins of extremophilic organisms that are only distantly related to organisms described so far. The PPM algorithm helps experimentalists to extract proteins and particularly enzymes with high confidence from databases with only limited annotation reliability, e.g., when SAGs of extremophiles are used.

The combination of orthogonal descriptors may also facilitate screening of other genomic data for proteins of interest, e.g., those resulting from metagenomic or metatranscriptomic sampling as well as from shotgun sequencing. For metagenomic sequences the most reliable functional annotations are achieved using homology-based approaches against publicly available reference sequence databases including GO. Recently, it was recommended for metagenomic data to run a motif-based analysis (e.g., using PROSITE-IDs) in parallel to the homology-based functional prediction (Prakash and Taylor, 2012). The PPM algorithm provides an example using this approach. However, since the PPM algorithm was developed to minimize the number of false positive hits when experimentalists search genomic databases for proteins of interest and we expect also for metagenomic data that the increased reliability of genes identified by this algorithm will be it's main advantage.

The publicly available scripts used in this study (i) AutoTECNo, (ii) PPM processor in combination with (iii) the INDIGO data warehouse are powerful tools, with a minimalistic character to keep handling of extreme large datasets simple. The PPM algorithm will facilitate experimental characterization of extremophilic proteins and therefore help to increase the general 
understanding of life at extreme conditions and exploiting its biotechnological potential. The enzymes identified in this study will be the first of many proteins on this path.

\section{ACKNOWLEDGMENTS}

We gratefully acknowledge financial support by the Saudi Economic and Development Company (SEDCO) Research Excellence Award "The Deep-Sea Brine Pools of the Red Sea: From Novel Extreme Organisms to Commercial Applications" and the King Abdullah University of Science and Technology (KAUST) faculty baseline funding (Jörg Eppinger, Ulrich Stingl, and Vladimir Bajic). We thank Prof. James Gregory Ferry from the Department of Biochemistry and Molecular Biology, Pennsylvania State University, for helpful discussion leading to identification of the $\gamma$ CA candidate genes described in this article. We are grateful for the valuable help of the scientists and crew on board RV Aegeo (3rd KAUST/WHOI Red Sea Expedition). We thank the Coastal and Marine Resources Core Lab (CMRC) of the King Abdullah University of Science and Technology for support and technical assistance and the Computational Bioscience Research Center (CBRC) computer cluster administration team, especially Allan Anthony Kamau for their help in making computational resources available for this work.

\section{SUPPLEMENTARY MATERIAL}

The Supplementary Material for this article can be found online at: http://www.frontiersin.org/journal/10.3389/fmicb. 2014.00134/abstract

\section{REFERENCES}

Alain, K., and Querellou, J. (2009). Cultivating the uncultured: limits, advances and future challenges. Extremophiles 13, 583-594. doi: 10.1007/s00792-0090261-3

Alam, I., Antunes, A., Kamau, A. A., Kalkatawi, M., Stingl, U., and Bajic, V. B. (2013). INDIGO-INtegrated Data Warehouse of MIcrobial GenOmes with examples from the red sea extremophiles. PLoS ONE 8:e82210. doi: 10.1371/journal.pone.0082210

Amann, R. I., Ludwig, W., and Schleifer, K.-H. (1995). Phylogenetic identification and in situ detection of individual microbial cells without cultivation. Microbiol. Rev. 59, 143-169.

Anschutz, P., and Blanc, G. (1995). Geochemical dynamics of the Atlantis II Deep (Red Sea): silica behavior. Mar. Geol. 128, 25-36. doi: 10.1016/00253227(95)00085-D

Antunes, A., Ngugi, D. K., and Stingl, U. (2011). Microbiology of the Red Sea (and other) deep-sea anoxic brine lakes. Environ. Microbiol. Rep. 3, 416-433. doi: 10.1111/j.1758-2229.2011.00264.x

Ashburner, M., Ball, C. A., Blake, J. A., Botstein, D., Butler, H., Cherry, J. M., et al. (2000). Gene ontology: tool for the unification of biology. The Gene Ontology Consortium. Nat. Genet. 25, 25-29. doi: 10.1038/ 75556

Backer, H., and Schoell, M. (1972). New deeps with brines and metalliferous sediments in the Red Sea. Nat. Phys. Sci. 240, 153-158. doi: 10.1038/physci240153a0

Bankevich, A., Nurk, S., Antipov, D., Gurevich, A. A., Dvorkin, M., Kulikov, A. S., et al. (2012). SPAdes: A new genome assembly algorithm and its applications to single-cell sequencing. J. Comput. Biol. 19, 455-477. doi: $10.1089 / \mathrm{cmb} .2012 .0021$

Bruneau, L., Jerlov, N. G., and Koczy, F. F. (1953). "Physical and chemical methods," in Reports of the Swedish Deep Sea Expedition, Vol. 3. (Elanders: Göteborg), 99-112.

Chistoserdova, L. (2010). Recent progress and new challenges in metagenomics for biotechnology. Biotechnol. Lett. 32, 1351-1359. doi: 10.1007/s10529-010-0306-9
Chitsaz, H., Yee-Greenbaum, J. L., Tesler, G., Lombardo, M.-J., Dupont, C. L., Badger, J. H., et al. (2011). Efficient de novo assembly of single-cell bacterial genomes from short-read data sets. Nat. Biotechnol. 29, 915-921. doi: 10.1038/nbt.1966

Cita, M. B. (2006). Exhumation of Messinian evaporites in the deep-sea and creation of deep anoxic brine-filled collapsed basins. Sediment. Geol. 188, 357-378. doi: 10.1016/j.sedgeo.2006.03.013

DasSarma, S., and Arora, P. (2001). "Halophiles" in Encyclopedia of Life Sciences, (London: John Wiley \& Sons, Ltd), 1-9. doi: 10.1038/npg.els.0000394

De Castro, E., Sigrist, C. J., Gattiker, A., Bulliard, V., Langendijk-Genevaux, P. S., Gasteiger, E., et al. (2006). ScanProsite: detection of PROSITE signature matches and ProRule-associated functional and structural residues in proteins. Nucleic Acids Res. 34, W362-W365. doi: 10.1093/nar/gkl124

Dolled-Filhart, M. P., Lee, M. Jr., Ou-Yang, C.-W., Haraksingh, R. R., and Lin, J. C.-H. (2013). Computational and bioinformatics frameworks for nextgeneration whole exome and genome sequencing. Sci. World J. 2013:730210. doi: 10.1155/2013/730210

Eder, W., Jahnke, L. L., Schmidt, M., and Huber, R. (2001). Microbial diversity of the brine-seawater interface of the Kebrit Deep, Red Sea, studied via 16S rRNA gene sequences and cultivation methods. Appl. Environ. Microbiol. 67, 3077-3085. doi: 10.1128/AEM.67.7.3077-3085.2001

Emery, K., Hunt, J., and Hays, E. (1969). "Summary of hot brines and heavy metal deposits in the Red Sea," in Hot Brines and Heavy Metal Deposits in the Red Sea, eds E. T. Degens, and D. A. Ross (Berlin: Springer Verlag), 557-571. doi: 10.1007/978-3-662-28603-6_50

Faber, E., Botz, R., Poggenburg, J., Schmidt, M., Stoffers, P., and Hartmann, M. (1998). Methane in Red Sea brines. Org. Geochem. 29, 363-379. doi: 10.1016/S0146-6380(98)00155-7

Garber, M., Grabherr, M. G., Guttman, M., and Trapnell, C. (2011). Computational methods for transcriptome annotation and quantification using RNA-seq. Nat. Methods 8, 469-477. doi: 10.1038/nmeth.1613

Gurvich, E. (2006). Metalliferous sediments of the world ocean: fundamental theory of deep-sea hydrothermal sedimentation. Heidelberg: Springer Berlin Heidelberg.

Hartmann, M., Scholten, J. C., Stoffers, P., and Wehner, F. (1998). Hydrographic structure of brine-filled deeps in the Red Sea-new results from the Shaban, Kebrit, Atlantis II, and Discovery Deep. Mar. Geol. 144, 311-330. doi: 10.1016/S0025-3227(97)00055-8

Horner, D. S., Pavesi, G., Castrignanò, T., De Meo, P. D. O., Liuni, S., Sammeth, M., et al. (2010). Bioinformatics approaches for genomics and post genomics applications of next-generation sequencing. Brief. Bioinformatics 11, 181-197. doi: 10.1093/bib/bbp046

Hovland, M., Kuznetsova, T., Rueslåtten, H., Kvamme, B., Johnsen, H. K., Fladmark, G. E., et al. (2006). Sub-surface precipitation of salts in supercritical seawater. Basin Res. 18, 221-230. doi: 10.1111/j.1365-2117.2006.00290.x

Hugenholtz, P., and Tyson, G. W. (2008). Microbiology: metagenomics. Nature 455, 481-483. doi: 10.1038/455481a

Jeyakanthan, J., Rangarajan, S., Mridula, P., Kanaujia, S. P., Shiro, Y., Kuramitsu, S., et al. (2008). Observation of a calcium-binding site in the-class carbonic anhydrase from Pyrococcus horikoshii. Acta Crystallogr. D Biol. Crystallogr. 64, 1012-1019. doi: 10.1107/S0907444908024323

Johnson, M., Zaretskaya, I., Raytselis, Y., Merezhuk, Y., McGinnis, S., and Madden, T. L. (2008). NCBI BLAST: a better web interface. Nucleic Acids Res. 36, W5-W9. doi: 10.1093/nar/gkn201

Kanehisa, M., and Goto, S. (2000). KEGG: kyoto encyclopedia of genes and genomes. Nucleic Acids Res. 28, 27-30. doi: 10.1093/nar/28.1.27

Karan, R., Kumar, S., Sinha, R., and Khare, S. (2012). "Halophilic microorganisms as sources of novel enzymes," in Microorganisms in Sustainable Agriculture and Biotechnology, eds T. Satyanarayana, B. Narain Johri, and A. Prakash (New Delhi: Springer), 555-579.

Karbe, L. (1987). "Hot brines and the deep sea environment," in Red Sea. Key Environments Series, eds A. J. Edwards, and S. M. Head (Oxford: Pergamon Press), 70-89.

Kisker, C., Schindelin, H., Alber, B. E., Ferry, J. G., and Rees, D. C. (1996). A lefthand beta-helix revealed by the crystal structure of a carbonic anhydrase from the archaeon Methanosarcina thermophila. EMBO J. 15, 2323.

Könneke, M., Bernhard, A. E., José, R., Walker, C. B., Waterbury, J. B., and Stahl, D. A. (2005). Isolation of an autotrophic ammonia-oxidizing marine archaeon. Nature 437, 543-546. doi: 10.1038/nature03911 
Kunin, V., Copeland, A., Lapidus, A., Mavromatis, K., and Hugenholtz, P. (2008). A bioinformatician's guide to metagenomics. Microbiol. Mol. Biol. Rev. 72, 557-578. doi: 10.1128/MMBR.00009-08

Lasken, R. S. (2007). Single-cell genomic sequencing using multiple displacement amplification. Curr. Opin. Microbiol. 10, 510-516. doi: 10.1016/j.mib.2007.08.005

Liszka, M. J., Clark, M. E., Schneider, E., and Clark, D. S. (2012). Nature versus nurture: developing enzymes that function under extreme conditions. Annu. Rev. Chem. Biomol. Eng. 3, 77-102. doi: 10.1146/annurev-chembioeng-061010114239

Lohse, M., Bolger, A. M., Nagel, A., Fernie, A. R., Lunn, J. E., Stitt, M., et al. (2012). RobiNA: a user-friendly, integrated software solution for RNA-Seq-based transcriptomics. Nucleic Acids Res. 40, W622-W627. doi: 10.1093/nar/gks540

Madigan, M., Martinko, J. M., and Parker, J. (2003). Brock Biology of Microorganisms. New Jersey: Pearson Education International.

Ollivier, B., Caumette, P., Garcia, J.-L., and Mah, R. A. (1994). Anaerobic bacteria from hypersaline environments. Microbiol. Rev. 58, 27-38.

Pabinger, S., Dander, A., Fischer, M., Snajder, R., Sperk, M., Efremova, M., et al. (2014). A survey of tools for variant analysis of next-generation genome sequencing data. Brief. Bioinform. 15, 256-278. doi: 10.1093/bib/bbs086

Peng, Y., Leung, H. C., Yiu, S. M., and Chin, F. Y. (2012). IDBA-UD: a de novo assembler for single-cell and metagenomic sequencing data with highly uneven depth. Bioinformatics 28, 1420-1428. doi: 10.1093/bioinformatics/bts174

Prakash, T., and Taylor, T. D. (2012). Functional assignment of metagenomic data: challenges and applications. Brief. Bioinformatics 13, 711-727. doi: 10.1093/bib/bbs033

Quevillon, E., Silventoinen, V., Pillai, S., Harte, N., Mulder, N., Apweiler, R., et al. (2005). InterProScan: protein domains identifier. Nucleic Acids Res. 33, W116-W120. doi: 10.1093/nar/gki442

Radivojac, P., Clark, W.T., Oron, T.R., Schnoes, A.M., Wittkop, T., Sokolov, A., et al., (2013). A large-scale evaluation of computational protein function prediction. Nat. Methods 10, 221-227. doi: 10.1038/nmeth.2340

Rohde, H., Qin, J., Cui, Y., Li, D., Loman, N. J., Hentschke, M., et al. (2011). Opensource genomic analysis of Shiga-toxin-producing E. coli O104: H4. N. Engl. J. Med. 365, 718-724. doi: 10.1056/NEJMoa1 107643

Ross, D. A. (1972). Red Sea hot brine area: revisited. Science 175, 1455-1457. doi: 10.1126/science.175.4029.1455

Saitou, N., and Nei, M. (1987). The neighbor-joining method: a new method for reconstructing phylogenetic trees. Mol. Biol. Evol. 4, 406-425.

Schnoes, A. M., Ream, D. C., Thorman, A. W., Babbitt, P. C., and Friedberg, I. (2013). Biases in the experimental annotations of protein function and their effect on our understanding of protein function space. PLoS Comput. Biol. 9:e1003063. doi: 10.1371/journal.pcbi.1003063

Schomburg, I., Chang, A., Placzek, S., Söhngen, C., Rother, M., Lang, M., et al. (2013). BRENDA in 2013: integrated reactions, kinetic data, enzyme function data, improved disease classification: new options and contents in BRENDA. Nucleic Acids Res. 41, D764-D772. doi: 10.1093/nar/gks1049

Searle, R. C., and Ross, D. A. (1975). A geophysical study of the Red Sea axial trough between $20.5^{\circ}$ and $22^{\circ}$ N. Geophys. J. Int. 43, 555-572. doi: 10.1111/j.1365246X.1975.tb00647.x
Sigrist, C. J., Cerutti, L., Hulo, N., Gattiker, A., Falquet, L., Pagni, M., et al. (2002). PROSITE: a documented database using patterns and profiles as motif descriptors. Brief. Bioinformatics 3, 265-274. doi: 10.1093/ bib/3.3.265

Sigrist, C. J. A., De Castro, E., Cerutti, L., Cuche, B. A., Hulo, N., Bridge, A., et al. (2013). New and continuing developments at PROSITE. Nucleic Acids Res. 41, D344-D347. doi: 10.1093/nar/gks1067

Škunca, N., Altenhoff, A., and Dessimoz, C. (2012). Quality of computationally inferred gene ontology annotations. PLoS Comput. Biol. 8:e1002533. doi: 10.1371/journal.pcbi.1002533

Smith, K. S., and Ferry, J. G. (2000). Prokaryotic carbonic anhydrases. FEMS Microbiol. Rev. 24, 335-366. doi: 10.1111/j.1574-6976.2000.tb00546.x

Smith, R. N., Aleksic, J., Butano, D., Carr, A., Contrino, S., Hu, F., et al. (2012). InterMine: a flexible data warehouse system for the integration and analysis of heterogeneous biological data. Bioinformatics 28, 3163-3165. doi: 10.1093/bioinformatics/bts577

Tamura, K., Stecher, G., Peterson, D., Filipski, A., and Kumar, S. (2013). MEGA6: molecular evolutionary genetics analysis version 6.0. Mol. Biol. Evol. 30, 2725-2729. doi: 10.1093/molbev/mst197

Triplet, T., and Butler, G. (2013). A review of genomic data warehousing systems. Brief. Bioinform. doi: 10.1093/bib/bbt031. [Epub ahead of print].

Watson, S. W., and Waterbury, J. B. (1969). "The sterile hot brines of the Red Sea," in Hot Brines and Recent Heavy Metal Deposits in the Red Sea, eds E. T. Degens, and D. A. Ross (New York, NY: Springer Berlin Heidelberg), 272-281. doi: 10.1007/978-3-662-28603-6_27

Yooseph, S., Sutton, G., Rusch, D. B., Halpern, A. L., Williamson, S. J., Remington, K., et al. (2007). The sorcerer II global ocean sampling expedition: expanding the universe of protein families. PLoS Biol. 5:e16. doi: 10.1371/journal.pbio. 0050016

Zuckerkandl, E., and Pauling, L. (1965). Evolutionary divergence and convergence in proteins. Evolving Genes Proteins 97, 97-166.

Conflict of Interest Statement: The authors declare that the research was conducted in the absence of any commercial or financial relationships that could be construed as a potential conflict of interest.

Received: 10 January 2014; accepted: 16 March 2014; published online: 07 April 2014. Citation: Grötzinger SW, Alam I, Ba Alawi W, Bajic VB, Stingl U and Eppinger $J$ (2014) Mining a database of single amplified genomes from Red Sea brine pool extremophiles-improving reliability of gene function prediction using a profile and pattern matching algorithm (PPMA). Front. Microbiol. 5:134. doi: 10.3389/fmicb. 2014.00134

This article was submitted to Extreme Microbiology, a section of the journal Frontiers in Microbiology.

Copyright (c) 2014 Grötzinger, Alam, Ba Alawi, Bajic, Stingl and Eppinger. This is an open-access article distributed under the terms of the Creative Commons Attribution License (CC BY). The use, distribution or reproduction in other forums is permitted, provided the original author(s) or licensor are credited and that the original publication in this journal is cited, in accordance with accepted academic practice. No use, distribution or reproduction is permitted which does not comply with these terms. 